\title{
Procrustes Problems for General, Triangular, and Symmetric Toeplitz Matrices
}

\author{
Juan Yang and Yuan-bei Deng \\ College of Mathematics and Econometrics, Hunan University, Changsha, China \\ Correspondence should be addressed to Yuan-bei Deng; ybdeng@hnu.edu.cn
}

Received 15 November 2013; Accepted 17 December 2013

Academic Editor: Mohamad Alwash

Copyright (C) 2013 J. Yang and Y.-b. Deng. This is an open access article distributed under the Creative Commons Attribution License, which permits unrestricted use, distribution, and reproduction in any medium, provided the original work is properly cited.

The Toeplitz Procrustes problems are the least squares problems for the matrix equation $A X=B$ over some Toeplitz matrix sets. In this paper the necessary and sufficient conditions are obtained about the existence and uniqueness for the solutions of the Toeplitz Procrustes problems when the unknown matrices are constrained to the general, the triangular, and the symmetric Toeplitz matrices, respectively. The algorithms are designed and the numerical examples show that these algorithms are feasible.

\section{Introduction}

Consider the constrained least-squares optimization problems:

$$
\min _{X \in P}\|A X-B\|_{F},
$$

where $A, B \in R^{n \times n}, P \subseteq R^{n \times n}$, and $\|\cdot\|_{F}$ denotes the Frobenius norm

$$
\|A\|_{F}=\sqrt{\operatorname{tr}\left(A^{T} A\right)}=\left(\sum_{i=1}^{m} \sum_{j=1}^{n} a_{i j}^{2}\right)^{1 / 2} .
$$

These problems have been investigated by a series of literatures. For instance, when $P=R^{n \times n}$, then $X=A^{+} B$ is a solution (refer to [1]), while the symmetric matrix solution case and orthogonal matrix solution case are analyzed in [2] and [3] respectively. And the symmetric positive (semi) definite least squares problem is discussed by Higham [4].

In this paper, the following Toeplitz least-squares problem are analyzed:

$$
\begin{array}{cl}
\min & \|A X-B\|_{F}^{2}, \\
\text { s.t. } & X \in \mathscr{T},
\end{array}
$$

where $A, B \in R^{n \times n}$ and $\mathscr{T} \subset R^{n \times n}$ are the set of Toeplitz matrices, and a matrix $T$ is called a Toeplitz matrix if its entries satisfy $T(i, j)=T(i+k, j+k)$, for all $i, j$, and $k$ with $0 \leqslant i+k \leqslant n-1,0 \leqslant j+k \leqslant n-1$,

$$
T=\left(\begin{array}{ccccc}
\alpha_{0} & \alpha_{1} & \alpha_{2} & \cdots & \alpha_{n-1} \\
\alpha_{-1} & \alpha_{0} & \alpha_{1} & & \vdots \\
\vdots & \ddots & \ddots & \ddots & \alpha_{2} \\
& & \ddots & \ddots & \alpha_{1} \\
\alpha_{-(n-1)} & \cdots & & \alpha_{-1} & \alpha_{0}
\end{array}\right)
$$

In this work, we discuss problem (3) in details. In Section 2, the general Toeplitz problem is discussed, and in Section 3, the triangular Toeplitz problem and the symmetric Toeplitz problem are discussed, while in Section 4, we give algorithms and numerical examples.

We give some definition and lemmas first.

Definition 1 (see [5]). Matrix $G_{n \times m}$ is called $\{1\}$ inverse matrix of $A_{m \times n}$ when $A G A=A$, and it can be denoted by $A^{-}$or $A\{1\}$.

Lemma 2 (see [5]). Let $A \in C_{r}^{m \times n}$; if there exist nonsingular matrices $P \in C^{n \times n}$ and $R \in C^{m \times m}$ such that

$$
R A P=\left[\begin{array}{cc}
I_{r} & \mathbf{0} \\
\mathbf{0} & \mathbf{0}
\end{array}\right],
$$


then the sufficient and necessary condition of $G \in A\{1\}$ is

$$
G=P\left[\begin{array}{ll}
I_{r} & U \\
V & W
\end{array}\right] R
$$

where $U \in C^{r \times(m-r)}, V \in C^{(n-r) \times r}$, and $W \in C^{(n-r) \times(m-r)}$.

Remark 3. When $U=\mathbf{0}, V=\mathbf{0}$, and $W=\mathbf{0}, G$ is denoted by $A_{*}^{-}$.

Lemma 4 (see [6]). A finite dimensional linear subspace of a normed linear space contains at least one point of minimum distance from a fixed point.

Lemma 5 (see [7]). Let $f: R^{n} \rightarrow R$ be a convex function continuously differentiable, and then any local minimum point is also a global minimum point. And $x^{*}$ is a solution of problem $\min f(x)$ if and only if $x^{*}$ satisfies $\nabla f\left(x^{*}\right)=0$, where $\nabla$ means the gradient of function $f$.

Lemma 6 (see [7]). The quadratic function $f(x)=$ $(1 / 2) X^{T} Q X+q^{T} X+C$ is convex if and only if $Q$ is positive semidefinite.

\section{The General Toeplitz Problem}

We transform problem (3) into the following equivalent form:

$$
\begin{gathered}
F \equiv \frac{1}{2}\|A X-B\|_{F}^{2}=\min , \\
X=\sum_{p=-(n-1)}^{(n-1)} \alpha_{p} G_{p},
\end{gathered}
$$

where $\alpha_{p} \in R, G_{p} \in R^{n \times n}$ are defined as

$$
\left(G_{p}\right)_{i j}=\left\{\begin{array}{ll}
1 & \text { if } j=i+p, \\
0 & \text { otherwise, }
\end{array} \quad p=-(n-1), \ldots,(n-1) .\right.
$$

The objective function $F$ can be rewritten as

$$
\begin{aligned}
F & =\frac{1}{2} \operatorname{tr}\left[(A X-B)^{T}(A X-B)\right] \\
& =\frac{1}{2} \operatorname{tr}\left(X^{T} A^{T} A X\right)+\left[-\operatorname{tr}\left(B^{T} A X\right)+\frac{1}{2} \operatorname{tr}\left(B^{T} B\right)\right] \\
& =F_{1}+F_{2} .
\end{aligned}
$$

The first term of the right side in (9) can be expressed as

$$
\begin{aligned}
F_{1} & =\frac{1}{2} \operatorname{tr}\left(X^{T} A^{T} A X\right) \\
& =\frac{1}{2} \operatorname{tr}\left(\left(\sum_{l=-(n-1)}^{(n-1)} \alpha_{l} G_{l}\right)^{T} A^{T} A\left(\sum_{k=-(n-1)}^{(n-1)} \alpha_{k} G_{k}\right)\right) \\
& =\frac{1}{2} \sum_{l, k=-(n-1)}^{(n-1)} \operatorname{tr}\left(G_{l}^{T} A^{T} A G_{k}\right) \alpha_{l} \alpha_{k} \\
& =\frac{1}{2} \sum_{l, k=-(n-1)}^{(n-1)} \frac{\operatorname{tr}\left(G_{l}^{T} A^{T} A G_{k}\right)+\operatorname{tr}\left(G_{k}^{T} A^{T} A G_{l}\right)}{2} \alpha_{l} \alpha_{k} \\
& =\frac{1}{2} y^{T} C y,
\end{aligned}
$$

where

$$
\begin{array}{r}
y^{T}=\left(\alpha_{-(n-1)}, \alpha_{-(n-2)}, \ldots, \alpha_{0}, \ldots, \alpha_{(n-1)}\right), \\
C=\left(C_{l k}\right)_{(2 n-1) \times(2 n-1)}, \\
C_{l k}=\frac{1}{2}\left(\operatorname{tr}\left(G_{l}^{T} A^{T} A G_{k}\right)+\operatorname{tr}\left(G_{k}^{T} A^{T} A G_{l}\right)\right), \\
l, k=-(n-1), \ldots,(n-1) .
\end{array}
$$

Also the second term of the right side in (9) can be expressed as

$$
\begin{aligned}
F_{2} & =-\operatorname{tr}\left(B^{T} A X\right)+\frac{1}{2} \operatorname{tr}\left(B^{T} B\right) \\
& =-\sum_{l=-(n-1)}^{(n-1)} \operatorname{tr}\left(B^{T} A G_{l}\right) \alpha_{l}+\frac{1}{2} \operatorname{tr}\left(B^{T} B\right) \\
& =-b^{T} y+\frac{1}{2} \operatorname{tr}\left(B^{T} B\right)
\end{aligned}
$$

where $b^{T}=\left(\operatorname{tr}\left(B^{T} A G_{-(n-1)}\right), \operatorname{tr}\left(B^{T} A G_{-(n-2)}\right), \ldots\right.$, $\operatorname{tr}\left(B^{T} A G_{(n-1)}\right)$.

It thus yields that

$$
F=\frac{1}{2} y^{T} C y-b^{T} y+\frac{1}{2} \operatorname{tr}\left(B^{T} B\right)
$$

Taking partial derivative on $F$ about $\alpha_{l}$ gives

$$
\begin{aligned}
\frac{\partial F}{\partial \alpha_{l}} & =\frac{\partial F_{1}}{\partial \alpha_{l}}+\frac{\partial F_{2}}{\partial \alpha_{l}} \\
& =\left(C_{l,-(n-1)}, C_{l,-(n-2)}, \ldots, C_{l,(n-1)}\right) y-\operatorname{tr}\left(B^{T} A G_{(l)}\right) \\
& =C_{l}^{T} y-b_{l}, \quad l=-(n-1), \ldots,(n-1) .
\end{aligned}
$$


By the first order necessary condition,

$$
\begin{gathered}
\frac{\partial F}{\partial \alpha_{-(n-1)}}=0, \\
\vdots \\
\frac{\partial F}{\partial \alpha_{(n-1)}}=0,
\end{gathered}
$$

we obtain

$$
C y=b .
$$

Therefore the following theorem holds.

Theorem 7. The solution of problem (3) exists, and its general form can be expressed as

$$
X=\sum_{l=-(n-1)}^{(n-1)} \alpha_{l} G_{l}
$$

where $\left(\alpha_{-(n-1)}, \alpha_{-(n-2)}, \ldots, \alpha_{0}, \ldots, \alpha_{(n-1)}\right)^{T}=y=C^{-} b+(I-$ $\left.C^{-} C\right) z$, for all $z \in R^{n}, C^{-}$means $\{1\}$ inverse of $C$.

Proof. Let $\mathscr{T}^{\prime}=\left\{T \in R^{n \times n} \mid T=A \sum_{p=-(n-1)}^{(n-1)} \alpha_{p} G_{p}\right\}$, where $\alpha_{p} \in R$, and then the subspace $\mathscr{T}^{\prime}$ is a normed linear space under Frobenius norm. It follows from Lemma 4 that there exists at least one matrix $T$ in $\mathscr{T}^{\prime}$ such that $\|A X-B\|_{F}^{2}=\min$. So, by the definition of $T$, there exists at least one scalar $\alpha^{T}=\left(\alpha_{-(n-1)}, \alpha_{-(n-2)}, \ldots, \alpha_{0}, \ldots, \alpha_{(n-1)}\right)$ constituting matrix $T$, which means we can rewrite $T$ as $T=A X$, where $X \in \mathscr{T}$. Therefore the solution of problem (3) exists.

It is apparent from (13) that the objective function $F$ is a quadratic function. Besides, by the following discussion, $F$ is also a convex function. Since $A^{T} A$ is positive semi-definite, it follows that, for any $X \in R^{n \times n}$, the expression of $F_{1}$ in (10) holds

$$
F_{1}=\frac{1}{2} y^{T} C y=\frac{1}{2} \operatorname{tr}\left(X^{T} A^{T} A X\right) \geqslant 0 .
$$

In other words, $C$ is a positive semidefinite matrix. Then according to Lemma $6, F$ is a convex function. Since $F$ is continuously differentiable, we then get that any solution of (16) is also a solution of (7) by Lemma 5. Clearly, the solution of linear system of (16) is

$$
y=C^{-} b+\left(I-C^{-} C\right) z, \quad \forall z \in R^{2 n-1}
$$

(see [5]). The required solution $X$ of problem (7) is then given by $X=\sum_{l=-(n-1)}^{(n-1)} \alpha_{l} G_{l}$, where $y=\left(\alpha_{-(n-1)}, \alpha_{-(n-2)}\right.$, $\left.\ldots, \alpha_{0}, \ldots, \alpha_{(n-1)}\right)^{T}$. The theorem follows by the equivalence of problem (3) and problem (7).

Theorem 8. Problem (3) has a unique solution if and only if $C$ has full rank. In this case, the unique solution is

$$
X=\sum_{l=-(n-1)}^{(n-1)} \alpha_{l} G_{l}
$$

where $\left(\alpha_{-(n-1)}, \alpha_{-(n-2)}, \ldots, \alpha_{0}, \ldots, \alpha_{(n-1)}\right)^{T}=y=C^{-1} b$.
Proof (necessity). If $C$ has full rank, then the linear system of (16) has a unique solution $y=C^{-1} b$; therefore the solution $X$ of problem (7) is determined uniquely, so is problem (3).

Sufficiency follows from the conversely procedure of necessity.

Corollary 9. If A has full rank in column, then the solution of problem (3) is unique.

\section{The Triangular Toeplitz Problem and the Symmetric Toeplitz Problem}

In this section, we discuss the triangular Toeplitz problem and symmetric Toeplitz problem.

3.1. The Triangular Toeplitz Problem. By the upper triangular Toeplitz problem, as the definition of [6], we mean the minimization problem

$$
\begin{array}{cl}
\min & \|A X-B\|_{F}^{2} \\
\text { s.t. } & X \in \mathscr{T}_{u},
\end{array}
$$

where $A, B \in R^{m \times n}, \mathscr{T}_{u}$ are the subspace of upper triangular Toeplitz matrices, with elements of the general form

$$
X=\left(\begin{array}{ccccc}
\alpha_{1} & \alpha_{2} & \alpha_{3} & \cdots & \alpha_{n} \\
0 & \alpha_{1} & \alpha_{2} & & \vdots \\
\vdots & \ddots & \ddots & \ddots & \alpha_{3} \\
& & \ddots & \ddots & \alpha_{2} \\
0 & \cdots & & 0 & \alpha_{1}
\end{array}\right)
$$

We transform problem (21) into the following problem

$$
\begin{gathered}
F \equiv \frac{1}{2}\|A X-B\|_{F}^{2}=\min , \\
X=\sum_{p=1}^{n} \alpha_{p} H_{p}
\end{gathered}
$$

where

$$
\left(H_{p}\right)_{i j}=\left\{\begin{array}{ll}
1 & \text { if } j=i+p-1, \\
0 & \text { otherwise, }
\end{array} \quad p=1, \ldots, n .\right.
$$

The proof procedure is similar to that in problem (7). In this case, the related function $F$, the unknown $y$, the linear system of equations, and the matrix $C$ as well as the scalar $b$ can be expressed as

$$
\begin{gathered}
F=\frac{1}{2} y^{T} C_{u} y-b_{u}^{T} y+\frac{1}{2} \operatorname{tr}\left(B^{T} B\right), \\
C_{u} y=b_{u}, \quad y^{T}=\left(\alpha_{1}, \alpha_{2}, \ldots, \alpha_{n}\right), \quad C_{u}=\left(C_{l k}\right)_{n \times n}, \\
C_{l k}=\frac{1}{2}\left(\operatorname{tr}\left(H_{l}^{T} A^{T} A H_{k}\right)+\operatorname{tr}\left(H_{k}^{T} A^{T} A H_{l}\right)\right), \\
l, k=1,2, \ldots, n, \\
b_{u}=\left(\operatorname{tr}\left(B^{T} A H_{1}\right), \operatorname{tr}\left(B^{T} A H_{2}\right), \ldots, \operatorname{tr}\left(B^{T} A H_{n}\right)\right) .
\end{gathered}
$$


Therefore we obtain a conclusion on upper triangular Toeplitz problem as follows.

Theorem 10. The solution of problem (21) exists, and its general form can be expressed as

$$
X=\sum_{l=1}^{n} \alpha_{l} H_{l}
$$

where $\left(\alpha_{1}, \alpha_{2}, \ldots, \alpha_{n}\right)^{T}=y_{u}=C_{u}^{-} b_{u}+\left(I-C_{u}^{-} C_{u}\right) z$, for all $z \in R^{n}, C_{u}^{-}$means $\{1\}$ inverse of $C_{u}$.

Theorem 11. Problem (21) has a unique solution if and only if $C_{u}$ has full rank. In this case, the unique solution is

$$
X=\sum_{l=1}^{n} \alpha_{l} H_{l}
$$

where $\left(\alpha_{1}, \alpha_{2}, \ldots, \alpha_{n}\right)^{T}=C_{u}^{-1} b_{u}$.

Corollary 12. If A has full rank in column, then the solution of problem (21) is unique.

Similarly, we can solve the lower triangular Toeplitz problem.

3.2. The Symmetric Toeplitz Problem. The symmetric Toeplitz problem is the following minimization problem:

$$
\begin{array}{cl}
\min & \|A X-B\|_{F}^{2} \\
\text { s.t. } & X \in \mathscr{T}_{s},
\end{array}
$$

where $A, B \in R^{m \times n}, \mathscr{T}_{s}$ are the subspace of symmetric Toeplitz matrices, with elements of the general form:

$$
X=\left(\begin{array}{ccccc}
\alpha_{1} & \alpha_{2} & \alpha_{3} & \cdots & \alpha_{n} \\
\alpha_{2} & \alpha_{1} & \alpha_{2} & & \vdots \\
\alpha_{3} & \alpha_{2} & \ddots & \ddots & \alpha_{3} \\
\vdots & & \ddots & \ddots & \alpha_{2} \\
\alpha_{n} & \cdots & \alpha_{3} & \alpha_{2} & \alpha_{1}
\end{array}\right)
$$

We consider the following objective function:

$$
\begin{gathered}
F \equiv \frac{1}{2}\|A X-B\|_{F}^{2}=\min , \\
X=\sum_{p=1}^{n} \alpha_{p} Q_{p},
\end{gathered}
$$

where

$$
\left(Q_{p}\right)_{i j}=\left\{\begin{array}{ll}
1 & \text { if }|j-i|=p-1, \\
0 & \text { otherwise, }
\end{array} \quad p=1, \ldots, n .\right.
$$

In this case, the related function $F$, the unknown $y$, the linear system of equations, and the matrix $C$ as well as the scalar $b$ can be expressed as

$$
\begin{gathered}
F=\frac{1}{2} y^{T} C_{s} y-b_{s}^{T} y+\frac{1}{2} \operatorname{tr}\left(B^{T} B\right), \\
C_{s} y=b_{s}, \quad y^{T}=\left(\alpha_{1}, \alpha_{2}, \ldots, \alpha_{n}\right), \quad C_{s}=\left(C_{l k}\right)_{n \times n},
\end{gathered}
$$

$$
\begin{gathered}
C_{l k}=\frac{1}{2}\left(\operatorname{tr}\left(Q_{l}^{T} A^{T} A Q_{k}\right)+\operatorname{tr}\left(Q_{k}^{T} A^{T} A Q_{l}\right)\right), \\
l, k=1,2, \ldots, n, \\
b_{s}=\left(\operatorname{tr}\left(B^{T} A Q_{1}\right), \operatorname{tr}\left(B^{T} A Q_{2}\right), \ldots, \operatorname{tr}\left(B^{T} A Q_{n}\right)\right) .
\end{gathered}
$$

Therefore we obtain a conclusion about the symmetric Toeplitz problem.

Theorem 13. The solution of problem (28) exists, and its general form can be expressed as

$$
X=\sum_{l=1}^{n} \alpha_{l} Q_{l}
$$

where $\left(\alpha_{1}, \alpha_{2}, \ldots, \alpha_{n}\right)^{T}=y=C_{s}^{-} b_{s}+\left(I-C_{s}^{-} C_{s}\right) z$, for all $z \in R^{n}$, and $C_{s}^{-}$means $\{1\}$ inverse of $C_{s}$.

Theorem 14. Problem (28) has a unique solution if and only if $C_{s}$ has full rank. In this case, the unique solution is

$$
X=\sum_{l=1}^{n} \alpha_{l} Q_{l}
$$

where $\left(\alpha_{1}, \alpha_{2}, \ldots, \alpha_{n}\right)^{T}=y=C_{s}^{-1} b_{s}$.

Corollary 15. If A has full rank in column, then the solution of problem (28) is unique.

\section{Computation and Examples}

The derivation of general Toeplitz problem leads to the following computational algorithm.

Algorithm 16.

(1) Generate matrices $G_{i}(i=-(n-1), \ldots,(n-1))$ according to the definition in (7).

(2) Compute the matrix $C$ and scalar $b$ by formula (11) and (12).

(3) If $C$ is of full rank, then solving the linear system of (16) directly, to obtain the solution $y=C^{-1} b$; else calculate $C^{-}$, forming $y=C^{-} b+\left(I-C^{-} C\right) z$, for all $z \in R^{n}$. In general, let $U, V$ and $W$ in Lemma 2 equal to 0 , we can obtain one of $\{1\}$ inverse of $C$, that is $C_{*}^{-}$.

(4) Compute $X$ from $X=\sum_{l=-(n-1)}^{(n-1)} \alpha_{l} G_{l}$, where $\left(\alpha_{-(n-1)}\right.$, $\left.\ldots, \alpha_{(n-1)}\right)=y^{T}$.

The algorithms for other special Toeplitz problems can be designed similarly.

We mention that, in the beginning of this paper, solutions for that two instances are actually obtained based on Algorithm 16. We can give another example of Toeplitz problem with matrix $A$ of full rank in column as follows. According to Corollaries 9, 12, and 15, the solution is unique. 
Example 17. Consider the following

$$
\begin{aligned}
A & =\left(\begin{array}{cccc}
-2 & 2 & 0 & -1 \\
1 & 0 & 0 & 2 \\
2 & 2 & 1 & 3 \\
2 & -2 & -1 & -1 \\
-1 & -1 & 2 & 1
\end{array}\right), \\
B & =\left(\begin{array}{cccc}
0 & -2 & -3 & 2 \\
1 & -2 & -3 & 1 \\
0 & 0 & -3 & -3 \\
2 & 0 & 2 & 2 \\
3 & 2 & 0 & -3
\end{array}\right) .
\end{aligned}
$$

Using the algorithms mentioned above, computing the Toeplitz problems by Matlab, we obtain the following results.

The general Toeplitz matrix solution is

$$
X_{*}=\left(\begin{array}{cccc}
-0.40791 & -0.83796 & 0.080264 & -0.041414 \\
-0.55794 & -0.40791 & -0.83796 & 0.080264 \\
0.62335 & -0.55794 & -0.40791 & -0.83796 \\
0.30656 & 0.62335 & -0.55794 & -0.40791
\end{array}\right) \text {. }
$$

The upper triangular Toeplitz matrix solution is

$$
X_{u}=\left(\begin{array}{cccc}
-0.37531 & -0.76827 & -0.069704 & -0.079890 \\
0.0 & -0.37531 & -0.76827 & -0.069704 \\
0.0 & 0.0 & -0.37531 & -0.76827 \\
0.0 & 0.0 & 0.0 & -0.37531
\end{array}\right)
$$

and the symmetric Toeplitz matrix solution is

$$
X_{s}=\left(\begin{array}{cccc}
-0.46064 & -0.64477 & 0.35659 & 0.27712 \\
-0.64477 & -0.46064 & -0.64477 & 0.35659 \\
0.35659 & -0.64477 & -0.46064 & -0.64477 \\
0.27712 & 0.35659 & -0.64477 & -0.46064
\end{array}\right)
$$

\section{Conclusions}

We have discussed the Toeplitz Procrustes problem including the general, triangular, and symmetric cases. After transforming the origin problem into a quadric form, we gain the general or unique solution with facility, by solving the linear system of equations.

\section{Acknowledgments}

The authors want to thank M. G. Eberle and M. C. Macle who have first proposed and studied the Toeplitz Procrustes problem. They also thank any referee for helpful suggestions or strengthening the theorems.

\section{References}

[1] G. H. Golub and C. F. van Loan, Matrix Computations, vol. 3, Johns Hopkins University Press, Baltimore, Md, USA, 1983.
[2] N. J. Higham, "The symmetric Procrustes problem," BIT. Numerical Mathematics, vol. 28, no. 1, pp. 133-143, 1988.

[3] N. J. Higham, "Computing the polar decomposition-with applications," Society for Industrial and Applied Mathematics. Journal on Scientific and Statistical Computing, vol. 7, no. 4, pp. 1160$1174,1986$.

[4] N. J. Higham, "Computing a nearest symmetric positive semidefinite matrix," Linear Algebra and its Applications, vol. 103, pp. 103-118, 1988.

[5] X. C. He and Y. W. Sun, Guangyi ni Juzhen Yinlun, Jiangsu Science and Technology Press, Nanjing, China, 1991.

[6] E. W. Cheney, Introduction to Approximation Theory, McGrawHill Book, New York, NY, USA, 1966.

[7] L. D. Hui and T. X. Qiao, Numerical Optimization, Science press, Beijing, China, 2006. 


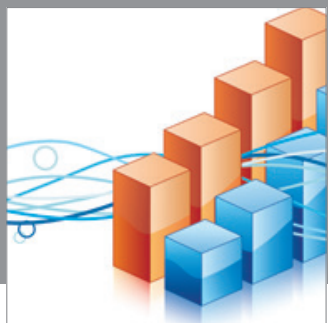

Advances in

Operations Research

mansans

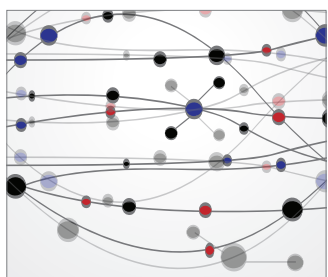

The Scientific World Journal
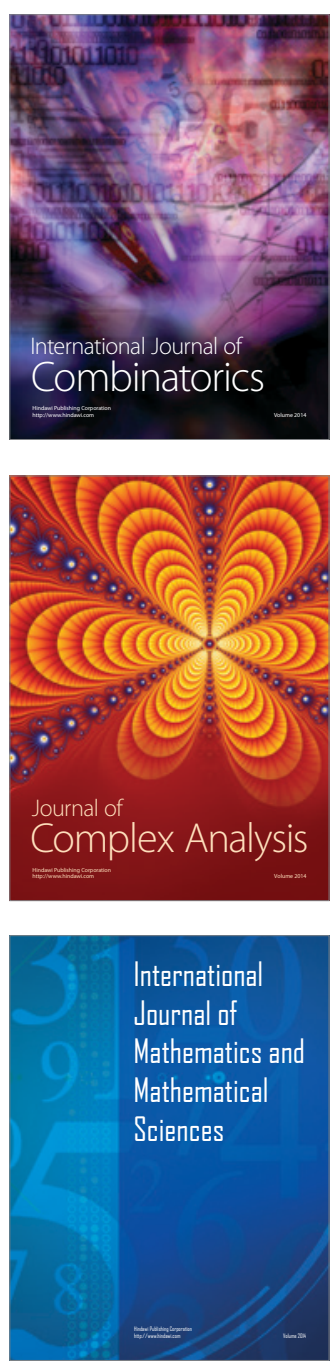
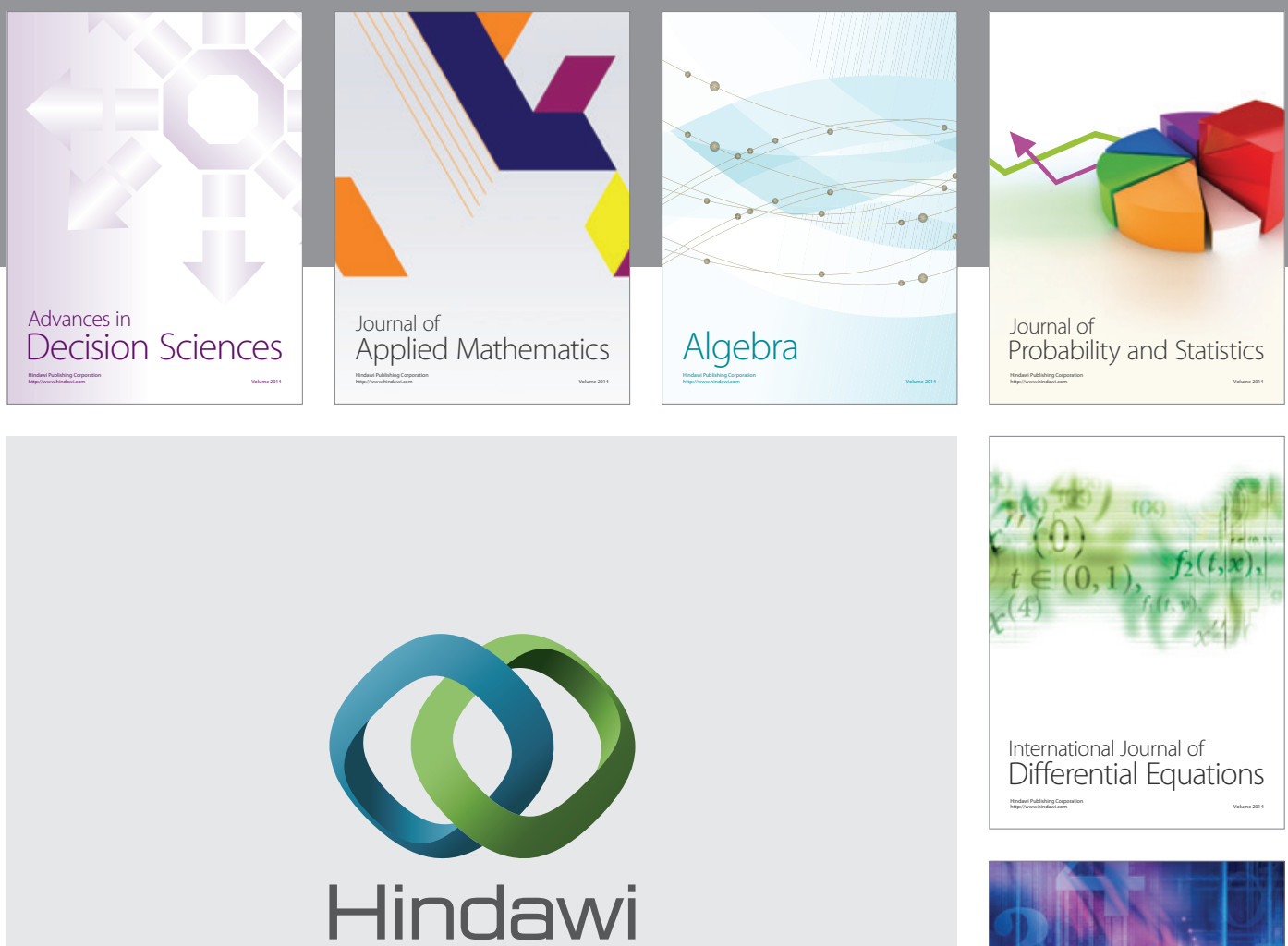

Submit your manuscripts at http://www.hindawi.com
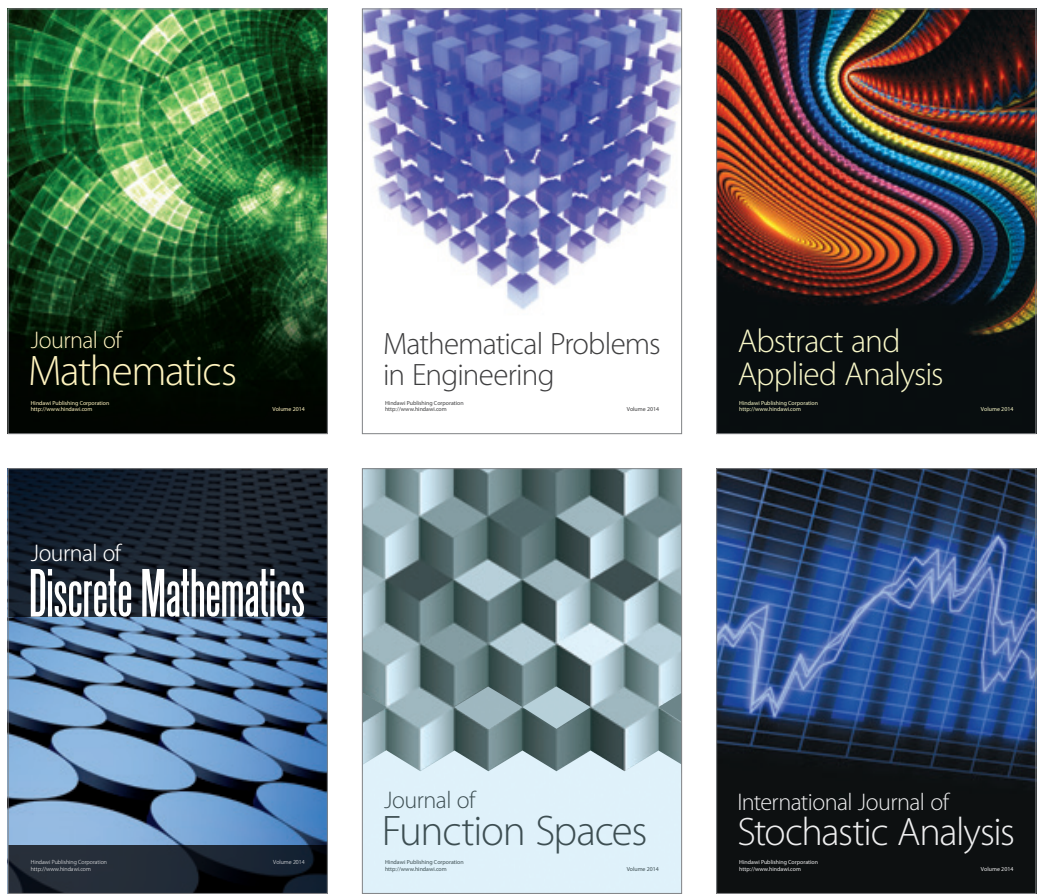

Journal of

Function Spaces

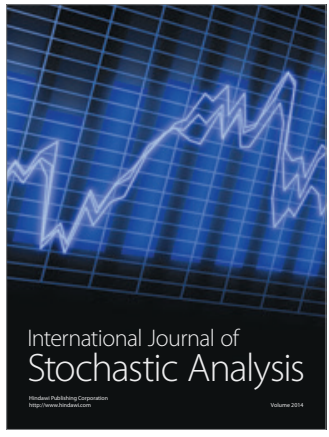

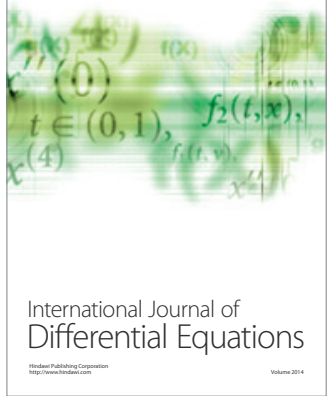
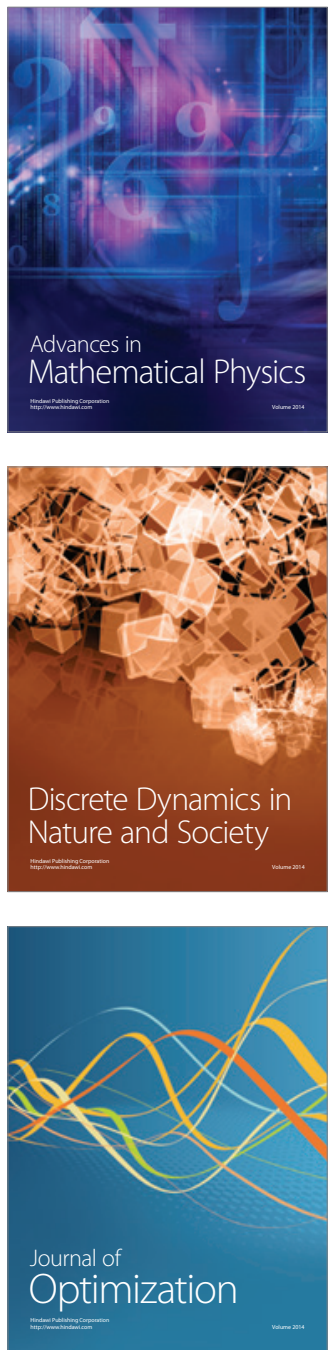